\title{
Optimisation of costs and carbon savings in relation to the economic dispatch problem as associated with power system operation
}

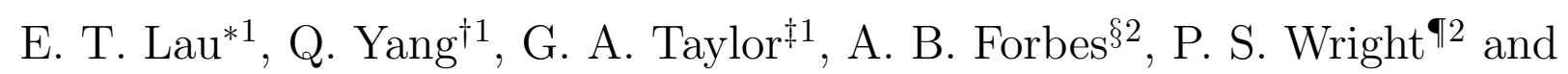
V. N. Livina ${ }^{\| 2}$

${ }^{1}$ Brunel University London, Kingston Lane, Uxbridge, UB8 3PH, UK ${ }^{2}$ National Physical Laboratory, Hampton Road, Teddington, Middlesex, TW11 0LW, UK

\begin{abstract}
In this paper, the costs and carbon savings in the Economic Dispatch (ED) problem of the power system operation are optimised. Energy demands and generation are forecast and assimilated using Ensemble Kalman Filter (EnKF). Optimisation is performed using the Ensemble-based Closed-Loop Production Optimisation Scheme (EnOpt). The real energy parameters of thermal units with green generators (wind farm) are used to test the methodology. The ability of the EnKF to predict, and the robustness of the EnOpt to optimise costs and the resultant carbon emissions are demonstrated. The proposed approach addresses the complexity and diversity of the power system and may be implemented in operational conditions of energy suppliers.
\end{abstract}

Keywords - Economic Dispatch, Carbon Savings, Ensemble Optimisation, Ensemble Kalman Filter

\footnotetext{
*eng.lau@brunel.ac.uk

${ }^{\dagger}$ qingping.yang@brunel.ac.uk

$\ddagger$ gareth.taylor@brunel.ac.uk

§alistair.forbes@npl.co.uk

^paul.wright@npl.co.uk

"|valerie.livina@npl.co.uk
} 


\section{Nomenclature}

\section{Acronyms}

BAU Business-as-usual

BMRS Balancing mechanism reporting system

ED Economic dispatch

EnKF Ensemble Kalman filter

EnOpt Ensemble-based closed-loop production optimisation scheme

$\mathrm{HH} \quad$ Half hourly

\section{Indexes}

$\alpha_{\lambda}$

$\lambda$

$i$

j

$k$

$t$

$t_{k}$

\section{Parameters}

$\lambda_{\max }$

$C_{\max , i}$

$d$

$E_{\max , i}$

$E_{\min , i}$

$h_{\max , i}$

m

$N_{e}$

$N_{i}$

$N_{k}$

$N_{t}$

$N_{x}$

$N E$

$N G$

$N W$

$W_{\text {max }, i}$

$\mathcal{E}_{\max , i}$

\section{Variables}

$\overline{\mathbf{x}_{\lambda}}$

$\overline{\mathcal{C}\left(\mathbf{x}_{\lambda}, Y^{u}\right)}$

$\mathbf{x}$

$\mathbf{x}_{\lambda}$

$\mathbf{x}_{\lambda+1}$

$\mathbf{x}_{\lambda, j}$
Tuning parameter that determines step size

Iteration index

Generating fuel index

Ensemble member index

Index for data times in EnOpt model

Time step index

Time step at data times $k$ in EnOpt model

Maximum iteration step

Maximum fuel cost at $i$ th generating unit

Model prediction

Maximum amount of energy generation for $i$ th generating unit

Minimum amount of energy generation for $i$ th generating unit

Maximum price penalty factor at $i$ th generating unit

Real parameters of energy data

Total number of ensemble members

Total number of fuels or generating units

Total number of data times for EnOpt model

Total number of time steps

Total number of control variables

Total number of generating units that contribute carbon emissions

Total number of thermal units

Total number of wind generators

Maximum amount of generation for $i$ th wind generator

Maximum emission cost at $i$ th generating unit

Mean value of control vector $\mathbf{x}$ at iteration $\lambda$

Mean value of objective function $\mathcal{C}\left(\mathbf{x}_{\lambda, j}, y_{j}^{u}\right)$

Vector of control variables

Control vector $\mathbf{x}$ at $\lambda$ th iteration

Updated control vector $\mathbf{x}$ at iteration $\lambda+1$

Realisation of control vector at iteration $\lambda$ and ensemble $j$ 


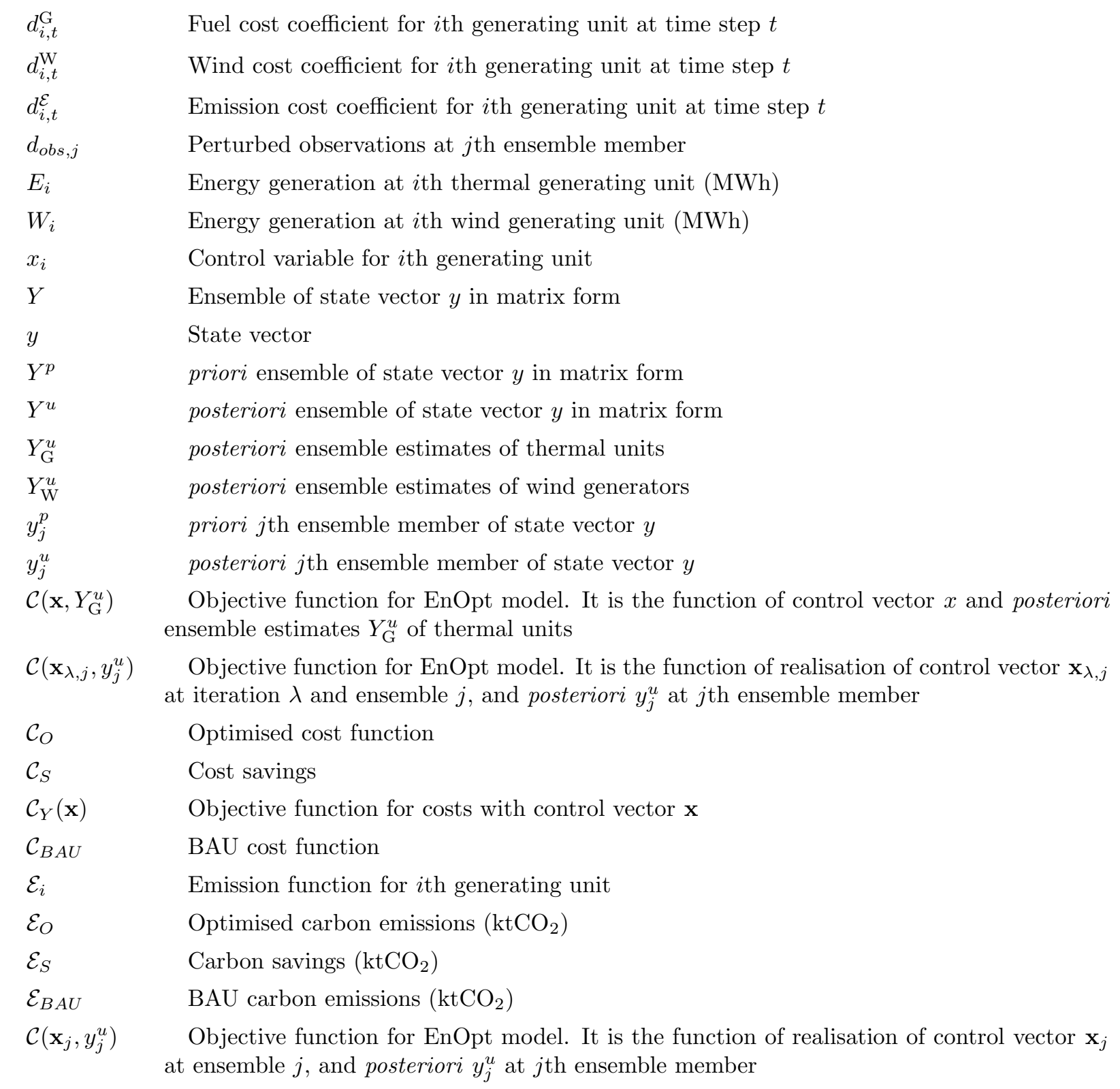

\section{Introduction}

In a power system, the grid operator aims to appropriately tune power flows with minimal systems losses [Crow, 2009]. However, the tuning of parameters in randomised manner without optimal planned strategy may increase the power operating costs, and also the environmental effects due to unnecessary firing-up of power plants. Instead of randomly tuning the parameters without proper arrangements and strategies, the optimisation of parameters through a selected objective function is required [Crow, 2009, Zhu, 2009]. The objective function is formulated to optimise operational strategies such as generating costs, reservoir production level, and system losses. With the increasing need to optimise power generation, the economical dispatch (ED) models were introduced. Due to the environmental concerns the ED problem has been undergoing major enhancement addressing the reduction of carbon emissions. Such enhancement is needed due to the legislation on the 2050 low carbon economy that requires the reduction of emissions by $80 \%$ below 1990 levels, with $40 \%$ reduced 
emissions by 2030 and $60 \%$ by 2040 [DG Clima, 2016]. The legislation by DG Clima [2016] requires the implementable and affordable participation of all sectors in the transition to low-carbon economy.

These legislations have led to implementation of various low carbon energy plans, with rising need to quantify the environmental impacts. One of the earlier modelling frameworks that addresses this is the UK MARKAL-Macro developed by Strachan and Kannan [2008]. The model predicts the aggregated energy demand response and technological change through the 2007 UK Energy White Paper policy framework. It provides the quantification of cost-economical implications due to the long-term decarbonisation strategies.

In the present paper, the novel modelling approach focusing on the quantification of costs and carbon emissions is proposed in the electricity generation which optimises costs and carbon savings. The approach is based on the ensemble Kalman filter (EnKF) combined with optimisation using ensemble-based closed-loop production (EnOpt) algorithm. The framework integrates the electrical data from generators (green and non-green energy) predicted and assimilated by EnKF and optimised by EnOpt, with operational constraints in a power system. In principle, this framework may be implemented at the level of the UK National Grid (the transmission operator) in collaboration with energy suppliers and distribution network operators.

The paper is organised as follows. Section 2 outlines modelling of energy systems, reviews the carbon factors, emissions and savings. The later includes the review of uncertainties in the power system and the introduction of the EnKF application. The review of the ED problem is also performed, along with the earlier optimisation technique in the power system and further introduces the EnOpt application. Section 3 presents the methodology for the ensemble assimilation and optimisation of costs and carbon savings. Section 4 presents the case study of the ED problem using the formulated EnKF and EnOpt algorithm. Section 5 discusses the numerical simulation results. Section 6 concludes.

\section{Modelling of power systems}

\subsection{General modelling approaches in power system}

The power system modelling is expanded substantially in order to mitigate the negative impact towards the environment, where the modelling includes the optimisation of the power system in the area of linear and nonlinear problems [Crow, 2009, Rau, 2009]. The general power system modelling approaches include the economic dispatch (ED), optimal power flow, unit commitment, and optimal load shedding [Zhu, 2009, Rau, 2009]. In this paper, the ED problem is applied to optimise the costs and carbon savings of the renewable and non-renewable energy.

\subsection{Environmental impact of power generation}

The UK carbon factors (also known as carbon footprints) are calculated by the company Ricardo-AEA [Ricardo-AEA, 2015], with quality assurance performed by the Department for Environment, Food and Rural Affairs (DEFRA) [Hill et al., 2013] and the Department of Energy and Climate Change (DECC) [DECC, 2013]. The results are reported annually 
by DEFRA. The latest data are available in the form of Microsoft Excel spreadsheets on the website [DEFRA, 2015], where statistics are currently stored for the years 2002-2015.

Carbon factors for fuel type with uncertainty ranges are reported in the post-notes of the Parliamentary Office of Science and Technology [POSTnote 268, 2006, POSTnote 383, 2011] and Carbon Trust [Carbon Trust, 2012], in grams (or kilograms) of carbon dioxide $\left(\mathrm{CO}_{2}\right)$ equivalent per unit of energy $(\mathrm{kWh})$. Since generated energy is given in $\mathrm{kWh}$ and carbon factors in $\mathrm{kgCO}_{2} / \mathrm{kWh}$, carbon emissions can be estimated as $\mathrm{CO}_{2}$ equivalent for a given time period.

Carbon savings are defined as the difference between the business-as-usual (BAU) emissions and the modified/optimised emissions, which are obtained by multiplying energy by corresponding carbon factors.

\subsection{Uncertainties in power systems}

The complexity of a power network requires flexibility of participating nodes, with consumers and generators connecting and disconnecting from the grid depending on conditions and demand. The conventional measurements of energy generation according to fuel types have been reported at 5 and 30 minute temporal resolution. Due to the variable dynamics of energy generation, the short-term forecast and assimilation of energy generation incorporating the uncertainty estimation is necessary. This is where the EnKF plays a useful role.

The green generators are known to be intermittent, and therefore the contribution to the grid is uncertain, hence non-green generators have to provide back-up generation to balance the power output. In the case of high amount of green generation, excess amount of generated energy may be created due to limited available energy storage. Experimental storage solutions are being developed but not employed at the level of National Grid yet. Thus, the green energy is uncertain, and the excess can be dumped in the system due to the infrastructural constraints.

Several methodologies have been developed to address uncertainties in power systems. One can express uncertainties using levels of fuzziness (fuzzy programming), or the Monte Carlo sampling techniques, for instance, the Stochastic Approximation (SA) and Sample Average Approximation (SAA). Lin et al. [2011] developed a dynamic optimisation model for energy systems planning under uncertainty through the integration of interval-parameter, fuzzy and mixed integer programming techniques within a network energy system. Li et al. [2010] applied a multistage interval-stochastic integer linear programming method in modelling electric power systems under uncertainty.

Similarly, Chen et al. [2010b] developed a two-stage inexact programming method for estimation of carbon emissions under uncertain network conditions. Zhu et al. [2013] further extended Li et al. [2010], Chen et al. [2010b] by developing a mixed-integer programming method for modelling carbon emissions to address uncertainties (for instance, constraints of energy demand and supply balance).

However, the accuracy of fuzzy strategies is low: Albertos and Sala [1998] argued that fuzzy strategies do not works well in scenarios where high levels of precision and accuracy are required. Nemirovski et al. [2009] stated that the multidimensional expectation integral of the objective function cannot be computed with high accuracy.

In this paper, EnKF is applied to forecast and assimilate the uncertain realisations of modelled energy generation, which realistically approximate conditions of the energy system. EnKF was first introduced by Evensen [1994] and further developed and applied in 
various branches of science (see Evensen [2003, 1994] and references therein). As described in Evensen [2003], Nævdal et al. [2003], Jensen [2007], Altaf et al. [2014], the EnKF is a recursive filter based on a Monte-Carlo approach to generate an ensemble of model representations. An ensemble is a system representation based on random sampling of system distribution [Evensen, 2003]. The 'true' state of the model is approximated by the ensemble mean [Almendral-Vazquez and Syversveen, 2006]. The covariance matrix in EnKF is predicted (forecast) and analysed by using statistics of the ensemble [Jensen, 2007]. Detailed formulation of EnKF can be found in [Evensen, 2003, Jensen, 2007, Haugen et al., 2008]. EnKF is suitable for sequential data assimilation in high-dimensional nonlinear systems. Even a few ensemble members have the ability to demonstrate the large-scale covariance behaviour of the system [John and Mandel, 2008]. EnKF has been widely used for assimilation of the real-time production data, providing both the estimate of a model and the corresponding uncertainty, by keeping track of the whole ensemble [Begum, 2009, Chen et al., 2009, Jahangiri, 2012].

\subsection{Optimisation in power systems}

With the increasing need to optimise the energy generation costs, ED models were introduced. As stated by Zhu [2009], EDs aim to minimise the operating cost of power generators by optimising the power output in each generators (thermal units) under specific constraints. The basic ED only minimises fuel costs subject to operational constraints. With the advancement of technological innovations along with environmental concerns, improved EDs have been developed incorporating the security of supply, emissions reduction and renewable generation.

The security-of-supply constraints are introduced in the ordinary ED model incorporating the power balance and line overload prevention [Huang et al., 2012]. The emissions function can be further added to the ED problem. However, such addition may introduce the trade-offs between the cost and emissions. The priority of optimising costs rather than emissions may increase emissions in the energy system. Ramanathan [1994], Rajasomashekar and Aravindhababu [2012] presented a methodology that concludes emissions constraints by applying the weighted sum technique to translate the multi-objective function (costs and emissions) into a single objective function. Similarly, Senthil and Manikandan [2010], Subramanian and Ganesan [2010] converted a multi-objective function to a single optimisation problem using the price penalty factor approach.

In addition to the security and emission constraints, the ED problem can be integrated with the renewable energy generation. Hetzer et al. [2008] included overestimation and underestimation of the available wind energy factor into the ED model. In their model, a linear cost function was assumed for the wind energy. On the other hand, Li et al. [2014] introduced the mean-variance multi-objective ED problem (due to the difficulty in obtaining the weights) for generators.

The basic ED problem is to be combined with other factors such as the security-ofsupply constraints, emissions and renewable generation. This is to decrease/balance the trade-offs of optimisation in the power system. Power generators are to be evaluated and their production optimised along with integration of the renewable energy, network security and emissions constraints.

Wei et al. [2014] proposed a bi-level (government and grid operators) economical optimisation model that determines the optimal tax rate among power generating units, balances 
the carbon emissions and profits of the energy sectors. Chen et al. [2013] developed an inexact optimisation method for supporting the carbon emissions management in the energy system, by employing interval-parameter programming within a robust optimisation framework. The optimisation scenarios generate alternative decisions to mitigate the carbon emissions within the economic context. Most interestingly, Cui et al. [2012] presented a robust bi-level model that combined consumers and utility companies to optimise social welfare by incorporating a feedback system that acted as a global controller.

However, the environmental impact of the power system is still evaluated at a very crude level. In the context of real-time control operations, the carbon reduction target is mostly pre-conditioned by the needs of positive economic impacts (profitable investments subject to operational constraints). The accurate assessment of costs and emissions in power systems is extremely important, as evaluation of energy data solely in terms of costs does not guarantee the environmental safety across the power system. In addition, limited access to power system data and infrequent metering have also led to uncertain carbon impacts of the operating strategies employed. Therefore, an efficient optimisation model is needed that does not only guarantee the profitable economical return of investment in the ED problem, but also addresses the need to reduce the nationwide emissions while adhering to operational constraints.

To this end, a new robust energy modelling and optimisation methodology is proposed that minimises costs and carbon emissions of the ED problem. In addition, a closed-loop based feedback optimisation control is proposed for continuous updates and optimisations in the ED problem. The EnOpt,

a production optimisation model that combines the EnKF and optimisation that was initially developed for the SmartWell technologies in the oil industry [Chen et al., 2009], is applied in order to develop optimal operating strategies in optimising the ED problem.

EnKF forecasts and assimilates system state with respect to the historical production data. Such data assimilation aims to minimise the mismatch between the model prediction and the production data [Chen et al., 2009]. The resultant assimilation data from EnKF is further utilised in the EnOpt, which estimates expectation of the objective function based on the assimilated data along with optimised control constraints [Chen et al., 2009, Jahangiri, 2012, Nwaozo, 2006]. At the end of the EnOpt simulation, the best control settings are the optimal control variables that minimise the objective function [Chen et al., 2009, Jahangiri, 2012, Nwaozo, 2006]. EnOpt has been well established as the optimisation algorithm in searching for optimal operating strategy in reservoir management [Chen et al., 2009, Jahangiri, 2012, Chen et al., 2010a, Petvipusit, 2011]. EnOpt is applied in this paper in order to develop operating strategies in the ED problem that optimise both costs and carbon savings.

\section{Methodology}

\subsection{Carbon factors, carbon emissions and savings of energy gen- eration and consumption}

Energy carbon factors have units of kilograms of carbon dioxide equivalent $\left(\mathrm{eqCO}_{2}\right)$ per unit of energy. These units describe all greenhouse gases involved that have the same global warming potential as the amount of $\mathrm{CO}_{2}$. In energy industry, the majority of greenhouse 
gases are produced by power stations, and even green generators have carbon footprints, which are derived using the Life Cycle Assessment methodology [Convenant of Mayors, 2010]. Carbon emissions $\mathcal{E}$ can be generally estimated as $\mathrm{CO}_{2}$ equivalent for a given time period (Eq. (1)).

$\mathcal{E}=\sum_{i=1}^{N_{i}} \sum_{t=1}^{N_{t}} E_{i}(t) \cdot F_{i}$,

where $E_{i}$ denotes generated energy, $i$ is the fuel index, $t$ is the time index, $F_{i}$ is the carbon factor for fuels, $N_{i}$ is the total number of fuels, $N_{t}$ is the total number of time steps.

To determine carbon emissions, the carbon factors of the electricity generators with known uncertainties are used. The Monte Carlo method with uniformly distributed random numbers drawn from uncertainty intervals corresponding to fuel types (as described in POSTnote 268 [2006], POSTnote 383 [2011], Lau et al. [2014]) is applied.

Carbon savings $S$ are determined as the difference between the BAU $\mathcal{E}_{B A U}$ and the optimised $\mathcal{E}_{O}$ carbon emissions:

$\mathcal{E}_{S}=\mathcal{E}_{B A U}-\mathcal{E}_{O}$,

where $\mathcal{E}_{B A U}$ is obtained based on either input energy data or model simulator. Optimised carbon emissions $\mathcal{E}_{O}$ are obtained using the EnOpt algorithm.

\subsection{The ED optimisation problem}

Fuel cost function. The fuel cost function (of $i$ th generating unit) is commonly described in a quadratic form of the active energy output [Huang et al., 2012, Ramanathan, 1994, Rajasomashekar and Aravindhababu, 2012, Senthil and Manikandan, 2010, Subramanian and Ganesan, 2010].

$\mathcal{C}_{i}\left(E_{i, t}\right)=a_{i}+b_{i} E_{i, t}+c_{i} E_{i, t}^{2}$,

where $a, b$ and $c$ are the cost coefficients of the generating unit $i, E$ is the amount of generated energy by the $i$ th generator at time $t$. The unit of the fuel cost function $C$ is $£ / H H$. The HH scale is used to present the energy data in Smart grid.

Emission function. The emission function of the ED model is also commonly described in the quadratic form [Huang et al., 2012, Ramanathan, 1994, Rajasomashekar and Aravindhababu, 2012, Senthil and Manikandan, 2010, Subramanian and Ganesan, 2010], which is the same as in Eq. (3)

$\mathcal{E}_{i}\left(E_{i, t}\right)=d_{i}+e_{i} E_{i, t}+f_{i} E_{i, t}^{2}$,

where $d, e$ and $f$ are the emissions coefficients of the generating unit $i, E$ is the amount of generation from the $i$ th generator at time $t$. The unit of the emission function $\mathcal{E}$ is $\mathrm{ktCO}_{2} / \mathrm{HH}$.

Renewable function. In this paper, the wind energy is selected as the renewable energy that integrates with the ED model. As suggested by Hetzer et al. [2008], Geetha et al. [2015], the linear cost function of the wind energy model is as follows:

$\mathcal{C}_{\mathrm{W}, i}\left(W_{i, t}\right)=d_{i, t}^{\mathrm{W}} W_{i, t}$ 
where $d_{i, t}^{\mathrm{W}}$ is the coefficient of wind generation at $i$ th wind generator at time $t, W_{i, t}$ is the active energy generation of $i$ th wind generator at time $t$.

Objective function. The multiple objective functions from Eqs. (3) to (5) are converted into a single objective function, incorporating the maximum price penalty factor $h_{\max }$ in the emission function. Following the methodology Senthil and Manikandan [2010], Subramanian and Ganesan [2010], Geetha et al. [2015], Khan et al. [2015], the objective function of the ED problem is follows:

$\mathcal{C}=\sum_{i=1}^{N G} \sum_{t=1}^{N_{t}} \mathcal{C}_{i}\left(E_{i, t}\right)+h_{\max , i} \sum_{i=1}^{N E} \sum_{t=1}^{N_{t}} \mathcal{E}_{i}\left(E_{i, t}\right)+\sum_{i=1}^{N W} \sum_{t=1}^{N_{t}} \mathcal{C}_{\mathrm{W}, i}\left(W_{i, t}\right)$.

The first term refers to the fuel cost (Eq. (3)), the second term is the emission function (Eq. (4)), the final term is the wind function (Eq. (5)). Here $N G$ is the total number of thermal units, $N E$ is the total number of generating units that contribute carbon emissions, $N W$ is the total number of wind generators.

The maximum price penalty factor $h_{\max , i}$ is calculated as the ratio of the maximum of the fuel cost to the maximum emissions at the energy output.

$h_{\max , i}=\frac{\mathcal{C}_{\max , i}}{\mathcal{E}_{\max , i}}$

Based on the publicly available fuel and emission cost data from [DECC, 2012], cost coefficients $(a-f)$ are excluded in this case. The available data from [DECC, 2012] allows one to estimate the direct profile trends of fuel and emission costs. This eliminates the needs to compute the cost coefficients corresponding to individual generators. The Eq. (6) is further simplified taking into account the BAU costs as follows:

$\mathcal{C}_{B A U}=\sum_{i=1}^{N G} \sum_{t=1}^{N_{t}} d_{i, t}^{\mathrm{G}} E_{i, t}+h_{\max , i} \sum_{i=1}^{N E} \sum_{t=1}^{N_{t}} d_{i, t}^{\mathcal{E}} E_{i, t}+\sum_{i=1}^{N W} \sum_{t=1}^{N_{t}} d_{i, t}^{\mathrm{W}} W_{i, t}$

where $d_{i, t}^{\mathrm{G}}$ and $d_{i, t}^{\mathcal{E}}$ are the cost coefficients of fuel and emission cost corresponding to $i$ th generating units at time $t$.

Similarly, the optimised cost of generation based on the integration of control vector $\mathbf{x}$ corresponding to $i$ th generating units for the EnOpt algorithm is calculated as:

$\mathcal{C}_{O}=\sum_{i=1}^{N G} \sum_{t=1}^{N_{t}} d_{i, t}^{\mathrm{G}} E_{i, \mathbf{x}, t}+h_{\max , i} \sum_{i=1}^{N E} \sum_{t=1}^{N_{t}} d_{i, t}^{\mathcal{E}} E_{i, \mathbf{x}, t}+\sum_{i=1}^{N W} \sum_{t=1}^{N_{t}} d_{i, t}^{\mathrm{W}} W_{i, t}$.

DECC [2012] provides documentation with levelised electricity generation cost ranges where the direct cost coefficient data $\left(d_{i, t}^{\mathrm{G}}, d_{i, t}^{\mathcal{E}}\right.$ and $\left.d_{i, t}^{\mathrm{W}}\right)$ can be obtained. Additionally, the cost coefficients are obtained dynamically in different periods (on- and off-peak periods). These coefficients are profiled based on the bid-offer spread of the electricity market imbalance volume. The bid-offer price data is available in the portal maintained by Elexon [2015]. Documentation of Elexon [2014] and Investopedia [2014] provide the detailed explanation of bid and offer price and the distribution spread, where spreads are determined by liquidity, 
as well as supply and demand for a specific security. According to Investopedia [2014], the high liquidity (high bid price) will have very low spreads due to no major imbalance between supply and demand. On the other hand, if there is a significant imbalance and low liquidity, the bid-ask spread will significantly expand.

The bid-offer price spread is further applied as the main profiling of cost coefficients and is calculated as:

bid_offer $_{i, t}=\frac{\text { offer_price }_{i, t}-\text { bid_price }_{i, t}}{\text { offer_price }_{i, t}}$,

where $i$ refers to the thermal units and wind generators at time $t$.

Using Eq. (10), the updated cost coefficient for the ED problem can be calculated as:

$d_{i, t}^{\mathrm{G}}=d_{i, t}^{\mathrm{G} \prime}\left(1+\right.$ bid_offer $\left._{i, t}\right)$,

$d_{i, t}^{\mathcal{E}}=d_{i, t}^{\mathcal{E} \prime}\left(1+\right.$ bid_offer $\left._{i, t}\right)$,

$d_{i, t}^{\mathrm{W}}=d_{i, t}^{\mathrm{W} \prime}\left(1+\right.$ bid_offer $\left._{i, t}\right)$.

Values $d_{i, t}^{\mathrm{G} \prime}, d_{i, t}^{\mathcal{E} \prime}$ and $d_{i, t}^{\mathrm{W} \prime}$ are the standard mean of prior cost coefficients resulting from the Monte-Carlo simulation of the levelised electricity generation costs from the data [DECC, 2012].

Based on Eq. (9), as wind generation is uncertain, other conventional generators (thermal units and hydro) are used to balance the wind output. The wind energy is generally assumed to be uncontrollable in adjusting the required load factor. Hence, there is no associated control vector in the wind function. In the context of EnKF, the wind energy can be used for short-term forecast and assimilation of the wind energy data. The short-term forecast of wind-generated data is possible by using $\mathrm{HH}$ wind generation data from Elexon [2015], Balancing Mechanism Reporting Systems [2015]. On the other hand, the wind energy will be implemented as the BAU solution in this paper, and other generators will be used to balance the wind output.

The total cost savings due to the EnOpt simulation are calculated as:

$\mathcal{C}_{S}=\mathcal{C}_{B A U}-\mathcal{C}_{O}$

Similarly, the Eq. (2) can be used to determine the amount of emission savings by using the second term of the cost function in Eqs. (8) and (9).

Constraints. The optimisation procedure is subject to the generation capacity constraints, energy balance (electricity market balance) constraints, and the actual wind energy generation limits:

$$
\begin{aligned}
& E_{\min , i} \leq E_{i, \mathrm{x}} \leq E_{\max , i}, \\
& \sum_{i=1}^{N G} E_{i, \mathrm{x}}+\sum_{i=1}^{N W} W_{i}-E_{\mathrm{L}}-E_{\mathrm{D}}=0, \\
& 0 \leq W_{i} \leq W_{\max , i}
\end{aligned}
$$

where $E_{\mathrm{L}}$ refers to the energy losses and $E_{\mathrm{D}}$ is the total energy demand, $N G$ is the total number of thermal units, $N W$ is the total number of wind generators. According to Balancing Mechanism Reporting Systems [2015], as it is the normal routine to have breakdowns 
during energy generation by fuel type, any fuel type with negative values in the dataset is capped to zero of total positive generation in order to meet the energy demand. Additionally, as there is no minimum operating energy limit for wind generation, the minimum amount of wind generation is set as zero [Geetha et al., 2015]. According to Ramanathan [1994], the emission constraint is not needed to be computed as the optimality condition can be achieved by imposing the constraint of the energy balance and generator limit.

\subsection{EnKF method}

As described by Nævdal et al. [2003], EnKF provides short-term forecast and uses ensemble realisations of a model state and state updates that are acquired through the combination of 'true' model and predicted ensemble estimates. Within EnKF, the model state is forecast and assimilated based on the ensemble propagated with the Kalman update. The formulations of EnKF as described by Evensen [2003], Nævdal et al. [2003], Almendral-Vazquez and Syversveen [2006], Chen et al. [2009] and Jahangiri [2012] are given below (note that only the key equations are provided).

The EnKF comprises two main steps, forecast and analysis. In the forecast step, as the 'true' state is not always available, new ensemble is created based on the realisations in each of the model state through the model dynamics (simulator).

$y_{j}=y+w_{j}$

where $j$ indexes the ensemble member, $y$ is the state vector of the model simulator, $y_{j}$ is the resultant new formation of a set of ensemble through the prediction of the model state $y$ at ensemble member $j, w_{j}$ is the model process noise. As in line with Almendral-Vazquez and Syversveen [2006], the initial ensemble members of $y$ are sampled from a normal distribution with the zero mean and standard deviation.

Spread of the ensemble members of $y$ are further combined into a matrix $Y$ to denote the priori ensemble:

$Y=\left[y_{1}, y_{2}, \ldots, y_{j}, \ldots, y_{N_{e}}\right]$

where $N_{e}$ is the total number of ensemble members.

On the other hand, during the analysis step, new observations from the measurement sets are represented by another ensemble. In order to obtain consistent error propagation using the EnKF, the observations have to be considered as random variables [Nævdal et al., 2003]. This is accomplished by using the actual measurement (or whenever measurements are available) as the reference and the random measurement noise $(d)$ is added to the measurement to obtain the perturbed observations denoted by $d_{o b s, j}$ [Evensen, 2003, Nævdal et al., 2003, Jensen, 2007].

$d_{\mathrm{obs}, j}=d+v_{j}$

where $v_{j}$ is the measurement noise at $j$ th ensemble member.

Both $y$ in Eq. (14) and $d$ in Eq. (16) are perturbed with model error: the process error $w$ with zero mean and covariance $Q$ for $y$ and similarly, the measurement error $v$ with zero mean and covariance $R$ for $d$, i.e. values $w$ and $v$ are assumed to be drawn from Gaussian distributions as $w \sim N(0, Q)$ and $v \sim N(0, R)$. The errors are important in 
EnKF, because without them the system may be over-specified and no solutions resulting from EnKF propagations would be obtained [Jensen, 2007].

In Eq. (15), priori ensemble $\left(y_{j}^{p}\right)$ will be propagated using EnKF to obtain the new posteriori ensemble $\left(y_{j}^{u}\right)$ using the EnKF updating formula:

$y_{j}^{u}=y_{j}^{p}+C_{Y} H^{T}\left(H C_{Y} H^{T}+R\right)^{-1}\left(d_{o b s, j}-H y_{j}^{p}\right)$.

$C_{Y}$ is the covariance matrix of $y, R$ is the covariance matrix of the Gaussian measurement error $v, H$ is the measurement operator relating model variables to perturbed observations $d_{o b s, j}$ at $j$ th ensemble member allowing for $v_{j}$ [Evensen, 2003]. The product of $C_{Y} H^{T}$ is the cross-covariance between state variables and predicted observations. $H C_{Y} H^{T}$ is the autocovariance of the predicted observations. $d$ in this case corresponds to $H y_{j}^{p}$. The product of $C_{Y} H^{T}\left(H C_{Y} H^{T}+R\right)^{-1}$ provides the Kalman gain. $C_{Y}$ is formulated as follows:

$C_{Y} \approx \frac{1}{N_{e}-1}\left(Y^{p}-\overline{Y^{p}}\right)\left(Y^{p}-\overline{Y^{p}}\right)^{T}$,

where $Y^{P}$ denotes the matrix form of $y_{j}^{p}$.

At the end of the EnKF data assimilation, the posteriori ensemble of the system state shall converge towards the 'true' data. Therefore, the model states and parameters are updated at the analysis step, not at the forecast step.

\subsection{EnOpt method}

EnOpt is applied to minimise the objective function, which describes costs and carbon emissions, based on the propagated posteriori $\left(y_{j}^{u}\right)$ ensemble (Eq. (17)). The approaches as described by Chen et al. [2009], Jahangiri [2012], Nwaozo [2006] and Jafroodi and Zhang [2011] are given below. Ensembles of $y_{j}^{u}$ as $Y^{u}$ are denoted in matrix form.

The control vector $\mathbf{x}$ is introduced that integrates the energy data modelled at different control steps. The control vector $\mathbf{x}$ is formulated as follows:

$\mathbf{x}=\mathbf{k} \cdot\left[x_{1}, x_{2}, \ldots, x_{i}, x_{N_{x}}\right]$.

Here $i$ indexes the components of $\mathbf{x}$ and $\mathbf{k}$ is the smoothing coefficient. The $N_{x}$ is the total number of control variables. It is calculated as the product of the number of thermal units and the control steps.

Eq. (9) is used as the primary objective function of the ED simulator model. Realisations made in the EnKF earlier are further applied in the EnOpt optimisation module to optimise the energy generation:

$\mathcal{C}\left(\mathbf{x}, Y_{\mathrm{G}}^{u}\right)=\sum_{t=1}^{N_{t}} d_{\mathrm{G}}(t) Y_{\mathrm{G}}^{u}(\mathbf{x}, t)+h_{\max } \sum_{t=1}^{N_{t}} d_{\mathcal{E}}(t) Y_{\mathrm{G}}^{u}(\mathbf{x}, t)+\sum_{t=1}^{N_{t}} d_{\mathrm{W}}(t) Y_{\mathrm{W}}^{u}(t)$,

where $Y_{\mathrm{G}}^{u}$ is the posteriori ensemble estimates of thermal generating units and $Y_{\mathrm{W}}^{u}$ is the posteriori ensemble estimates of wind generators. $\mathrm{x}$ represents the control vector to be optimised. Both $Y_{\mathrm{G}}^{u}$ and $Y_{\mathrm{W}}^{u}$ are based on the resultant EnKF propagation using Eq. (17).

Since the EnOpt algorithm utilises realisations of $y_{j}^{u}$ with $N_{e}$ simulation runs, the augmented objective function from Eq. (20) can be denoted as

$\mathcal{C}_{Y}(\mathbf{x})=\frac{1}{N_{e}} \sum_{j=1}^{N_{e}} \mathcal{C}\left(\mathbf{x}_{j}, y_{j}^{u}\right)$ 
Here $N_{e}$ denotes the total number of ensemble members, $\mathcal{C}\left(\mathbf{x}_{j}, y_{j}^{u}\right)$ is based on realisations of $y_{j}^{u}$ with $N_{e}$ simulation runs only. The subscript $Y$ indicates that costs and emissions are optimised based on the simulator model updated in the EnKF [Chen et al., 2009]. The $y_{j}^{u}$ stays constant during the production optimisation process at specific time step while $\mathbf{x}$ is optimised. Therefore $\mathcal{C}_{Y}(\mathbf{x})$ is expressed as a function of $\mathbf{x}$ only.

The steepest descent method is applied to obtain the optimal $\mathbf{x}$ that minimises $\mathcal{C}_{Y}(\mathbf{x})$. The steepest descent is performed as follows:

$\mathbf{x}_{\lambda+1}=\frac{1}{\alpha_{\lambda}} C_{\mathbf{x}} C_{\mathbf{x}, \mathcal{C}_{Y}(\mathbf{x})}-\mathbf{x}_{\lambda}$,

where $\lambda$ denotes the iteration index, $\alpha_{\lambda}$ is the tuning parameter that determines the step size, $C_{\mathbf{x}}$ is the covariance matrix of $\mathbf{x}, C_{\mathbf{x}, \mathcal{C}_{Y}(\mathbf{x})}$ is cross-covariance between $\mathbf{x}$ and $\mathcal{C}_{Y}(\mathbf{x})$ and is formulated as follows:

$C_{\mathbf{x}, \mathcal{C}_{Y}(\mathbf{x})} \approx \frac{1}{N_{e}-1} \sum_{j=1}^{N_{e}}\left(\mathbf{x}_{\lambda, j}-\overline{\mathbf{x}_{\lambda}}\right)\left(\mathcal{C}\left(\mathbf{x}_{\lambda, j}, y_{j}^{u}\right)-\overline{\mathcal{C}\left(\mathbf{x}_{\lambda}, Y^{u}\right)}\right)$,

with

$\overline{\mathbf{x}_{\lambda}}=\frac{1}{N_{e}} \sum_{j=1}^{N_{e}} \mathbf{x}_{\lambda, j}, \quad \overline{\mathcal{C}\left(\mathbf{x}_{\lambda}, Y^{u}\right)}=\frac{1}{N_{e}} \sum_{j=1}^{N_{e}} \mathcal{C}\left(\mathbf{x}_{\lambda, j}, y_{j}^{u}\right)$.

\subsection{Implementation of EnKF and EnOpt}

In EnOpt, $\mathbf{x}$ is optimised at each $\lambda$ th iteration, whereas $y_{j}^{u}$ and $Y^{u}$ are kept constant at every particular time step $t_{k}$ (during production optimisation process only). $k\left(1,2, \ldots, N_{k}\right)$ is the index for data times with $N_{k}$ denoting the total number of data times for EnOpt model.

The EnKF and EnOpt implementation steps are as follows:

1. Set $k=0$. Initialise and generate ensemble $Y_{0}$ and $\mathbf{x}$.

2. Propagate $Y_{k}$ and $\mathbf{x}$ from $t_{k}$ to $t_{k+1}$ through the simulator model.

3. Apply EnKF to obtain $Y^{u}$ by updating $Y_{k}$ using Eq. (17). Set $k=k+1$.

4. Start optimisation for the EnOpt at $\lambda=1$, generate $\mathbf{x}_{1}$ and $\mathbf{x}_{1, j}, j=1,2, \ldots, N_{e}$.

(a) If $k=1$ ( $k$ is the first time step), $\mathbf{x}_{1, j}$ is generated in two steps. First, the mean of the control is sampled from uniform distribution with lower and upper bounds of thermal capacity constraints. Second, the control mean is further perturbed by adding Gaussian random number with zero mean $\left(N \sim\left(0, C_{\mathbf{x}}\right)\right)$.

(b) If $k \neq 1$ ( $k$ is not the first time step), $\mathbf{x}_{1}$ is set to $\mathbf{x}_{1}=\mathbf{x}$ in each realisations. The $\mathbf{x}_{1}$ is the control optimised at the previous data assimilation. Gaussian random number with zero mean $\left(N \sim\left(0, C_{\mathbf{x}}\right)\right)$ is added to $\mathbf{x}_{1}$ to form $\mathbf{x}_{1, j}$.

5. If $\lambda \neq 1$, Gaussian random number with zero mean $\left(N \sim\left(0, C_{\mathbf{x}}\right)\right)$ is added to form $\mathbf{x}_{\lambda}$ in each realisations. 
6. Run the optimisation model (Eq. (9)) and calculate $\mathcal{C}\left(\mathbf{x}, Y_{\mathrm{G}}^{u}\right)$ using Eq. (20), where $Y_{\mathrm{G}}^{u}$ refers to the ensemble representation of $Y_{k}^{u}$.

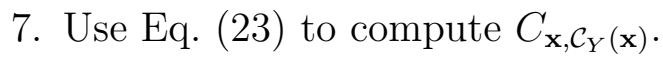

8. Compute $\mathbf{x}_{\lambda+1}$ using Eq. (22).

9. Evaluate $\mathcal{C}_{Y}\left(\mathbf{x}_{\lambda+1}\right)$ using Eq. (21) that require $N_{e}$ realisations.

10. If $\mathcal{C}_{Y}\left(\mathbf{x}_{\lambda+1}\right)<\mathcal{C}_{Y}\left(\mathbf{x}_{\lambda}\right)$, replace $\mathbf{x}_{\lambda}$ by $\mathbf{x}_{\lambda+1}$ and let $\lambda=\lambda+1$; otherwise, keep $\mathbf{x}_{\lambda}$, increase $\alpha_{\lambda}$ and proceed to step (8).

11. If stopping criteria is satisfied, set $\mathbf{x}=\mathbf{x}_{\lambda}$ and exit the optimisation loop; otherwise, repeat from step (5).

12. Repeat from step (2) until the end of the data assimilation $t$.

Stopping criteria include: (i) a maximum optimisation step $\lambda_{\max }$; (ii) unsuccessful search for $\alpha_{\lambda}$; (iii) $\alpha_{\lambda}$ is not allowed to increase more than twice; (iv) the relative iterative increase of the objective function is less than $1 \%$.

The obtained $\mathcal{E}$ in the second term of Eq. (9) is further used to represent the expectation of optimised carbon emissions $\mathcal{E}_{O}$ in Eq. (2). Similarly, the tabulated values of $\mathcal{C}_{O}$ at the end of EnOpt simulation (Eq. (20)) are used to compare with $\mathcal{C}_{B A U}$ (Eq. (8)). The cost saving in the ED problem is calculated using Eq. (12).

\section{Case study}

This section shows the case study of the ED problem using the EnKF and EnOpt algorithms as formulated in Sections 3.3 and 3.4.

\subsection{EnKF application}

In EnKF, the main goal is to estimate posteriori ensemble based on the energy generation. The EnKF needs to be initialised by providing model and input parameters for computation of the priori ensemble.

Variables of interest are collected into a state vector $y$ as the input of the model state:

$y=\left[\begin{array}{l}m \\ d\end{array}\right]$.

Eq. (25) also corresponds to the main input simulator model in Step 2 of the EnKF and EnOpt implementation (Section 3.5). In this case study, it is assumed that $m$ are real parameters of the historical energy data representing the thermal units and wind generators (known profiles of thermal units and wind generators) from the portal [Elexon, 2015, Balancing Mechanism Reporting Systems, 2015]. The available $m$ results in model prediction $d$ of the energy generation data for thermal units and wind generators. Parameters $m$ are adopted from the portal [Elexon, 2015, Balancing Mechanism Reporting Systems, 2015], and remain constant throughout the data assimilation. On the other hand, $d$ is the model prediction (actual measurement) of energy generation $E_{i, t}$ with real parameters $m$. The component 
$d$ in vector $y$ changes with data assimilation, incorporating the model errors in addition to $m$ at every time step.

The component $y$ is augmented as follows:

$y=\left[m_{1}, m_{2}, \ldots, m_{i}, E_{1}, E_{2}, \ldots, E_{i}, W_{1}, W_{2}, \ldots, W_{i}\right]^{T}$.

The $m$ component refers to real parameters of the thermal units and wind generators [Elexon, 2015, Balancing Mechanism Reporting Systems, 2015]. The components of $d$ consist of: 1) the predicted energy generation data for thermal units $\left.E_{1}, E_{2}, \ldots, E_{i} ; 2\right)$ the predicted wind energy generation $W_{1}, W_{2}, \ldots, W_{i}$.

The 'true' data of the energy generation from Elexon [2015], Balancing Mechanism Reporting Systems [2015] will be further predicted using ensemble representations (denoted as actual measurements $d$ ), with perturbed observations $\left(d_{o b s, j}\right)$ based on the perturbation of $d$.

Sets of $y$ are stored in matrix $Y$ (Eq. (15)) and are further denoted as priori ensemble $\left(y_{j}^{p}\right)$. The $y_{j}^{p}$ is further assimilated in the EnKF algorithm in order to obtain the new updated posteriori ensemble $\left(y_{j}^{p}\right)$ using Eq. (17).

\subsection{EnOpt application}

The component of control vector $\mathbf{x}$ in EnOpt contains energy generation output of thermal units that minimises costs and carbon emissions within the imposed constraints (Eq. (13)) for ED optimisation problem. The total number of control variables $N_{x}$ from Eq. (19) is calculated as the product of the number of thermal units and the control steps. By following the implementation step in Sections 3.4 and 3.5, the output variables - the optimised cost and carbon emissions based on the ED problem - can be obtained.

\section{Results and discussion}

\subsection{EnKF numerical simulation}

The EnKF short-term prediction (forecast) and assimilation are obtained based on the historical and real-time HH records of energy generation data Elexon [2015], Balancing Mechanism Reporting Systems [2015]. Five energy generation profiles for thermal units and one offshore wind generator are considered. Energy data in the beginning of spring season (01/03/2015) is adopted with the addition of model noises in order to forecast the total energy generation by the power plants. Since the historical and real-time HH records of energy generation are available, variable $y$ in Eq. (25) contributes to direct model predictions $(d)$ of the energy generation based on the real energy data $(m)$ of thermal units and wind generator.

The input data of energy generation is simulated using the modelled parameters in Eq. (25) for EnKF predictions. Various EnKF realisations are produced $\left(N_{e}=10,100,1000\right)$ through Eqs. (15) and (26) and propagated at every time steps. As in line with AlmendralVazquez and Syversveen [2006], the initial ensemble members of $y^{p}$ are drawn from a normal distribution with mean and standard deviation such that $N(0,20)$. Additionally, the model (process) error $w$ is sampled from $w \sim N(0,1)$. The measurement error, on the other hand, is sampled as $v \sim N(0,0.5)$. The assimilation is performed for 48 time steps $(24$ hours with $\mathrm{HH}$ interval). The ensemble corresponding to one day period in matrix $Y$ (Eq. (15)) is the 
prior $\left(y_{j}^{p}\right)$ ensemble. The ensemble is updated to form the posterior $\left(y_{j}^{u}\right)$ ensemble (Eq. (17)) and is stored in the matrix $Y^{u}$. Values $Y^{u}$ of energy generation allows the comparison of convergence in relation to the perturbed observations $d_{o b s, j}$. Quantity $d_{o b s, j}$ is generated by perturbing $d$.

The daily plot of the resultant energy generation (thermal units plus wind generators) and the EnKF assimilation of $Y^{u}$ with different realisations is shown in Fig. 1. The figure shows that the larger the ensemble size, the better $Y^{u}$ estimation converges towards the observation.

Additionally, the EnKF assimilation corresponding to thermal units and wind generation are performed. This is to examine the performance of EnKF in the assimilation of the individual units. Figs. 2 and 3 show different realisations of the diurnal EnKF'ed energy generation from thermal units and wind generators. The small ensemble size $\left(N_{e}=10\right)$ in Fig. 3 results in poor EnKF forecast and assimilation of wind data. Both simulations show that the larger the ensemble sizes, the lesser oscillation for the assimilation in EnKF. Consequently, the smaller the EnKF errors relatively to the observations.

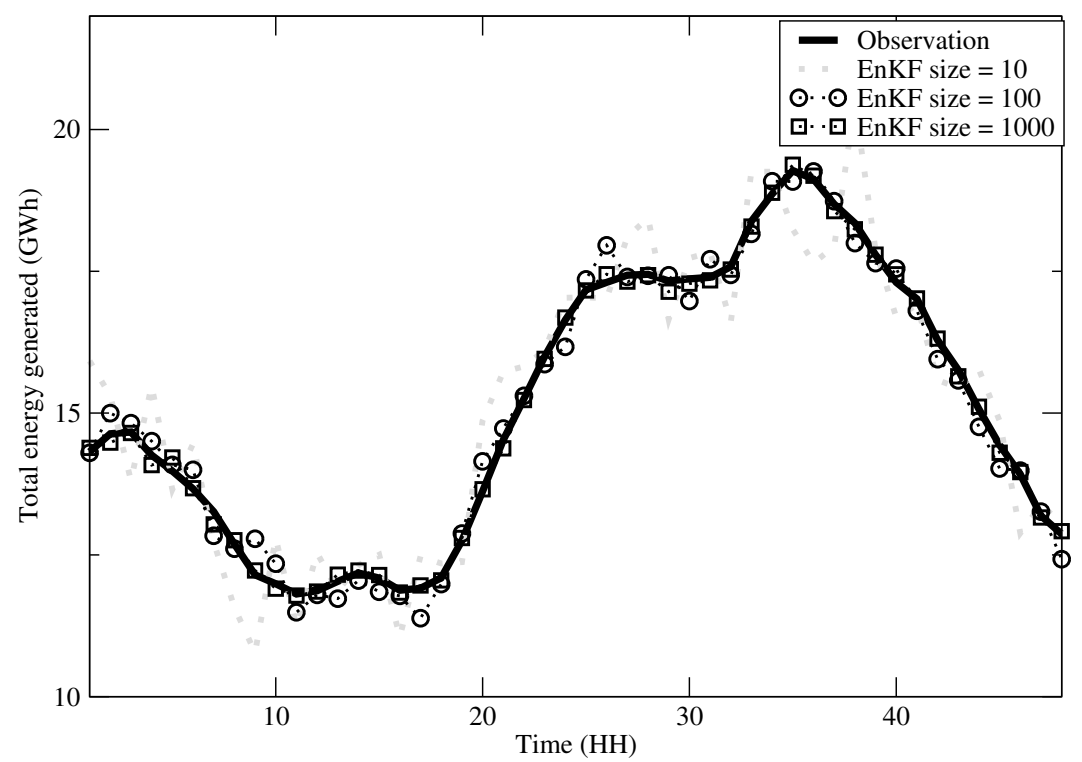

Figure 1: Total energy generation with different EnKF realisations. Generating fleets are thermal units and wind generators. 


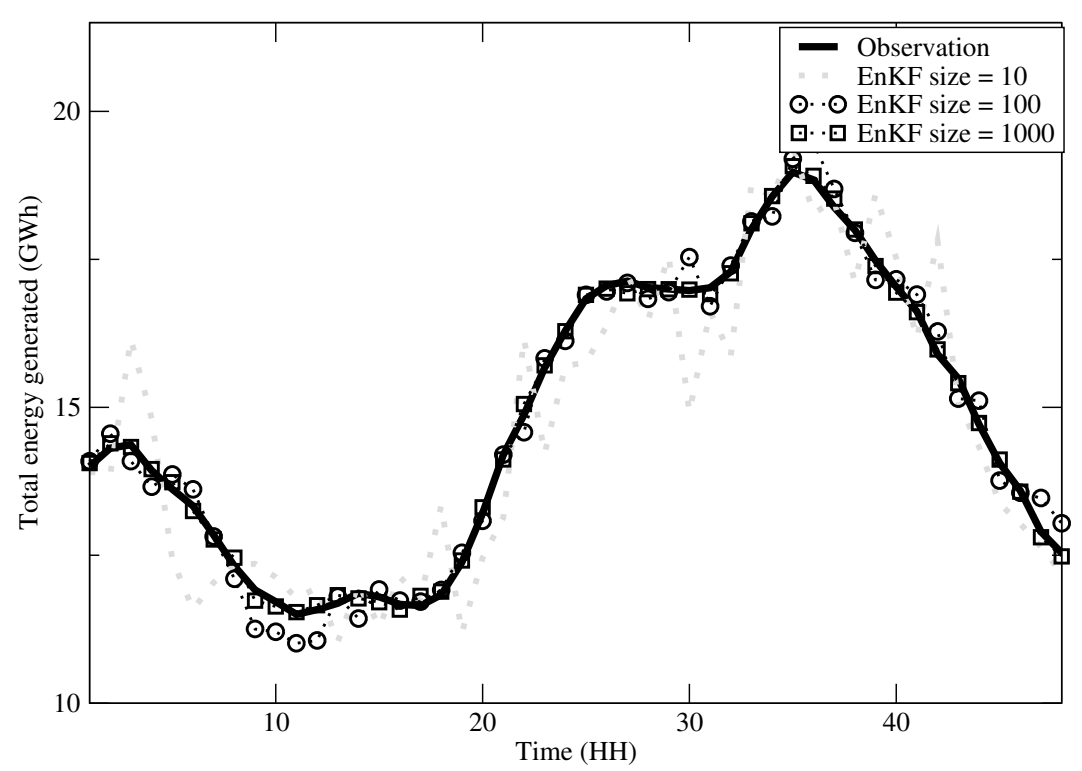

Figure 2: Total energy generation of thermal units with different EnKF realisations.

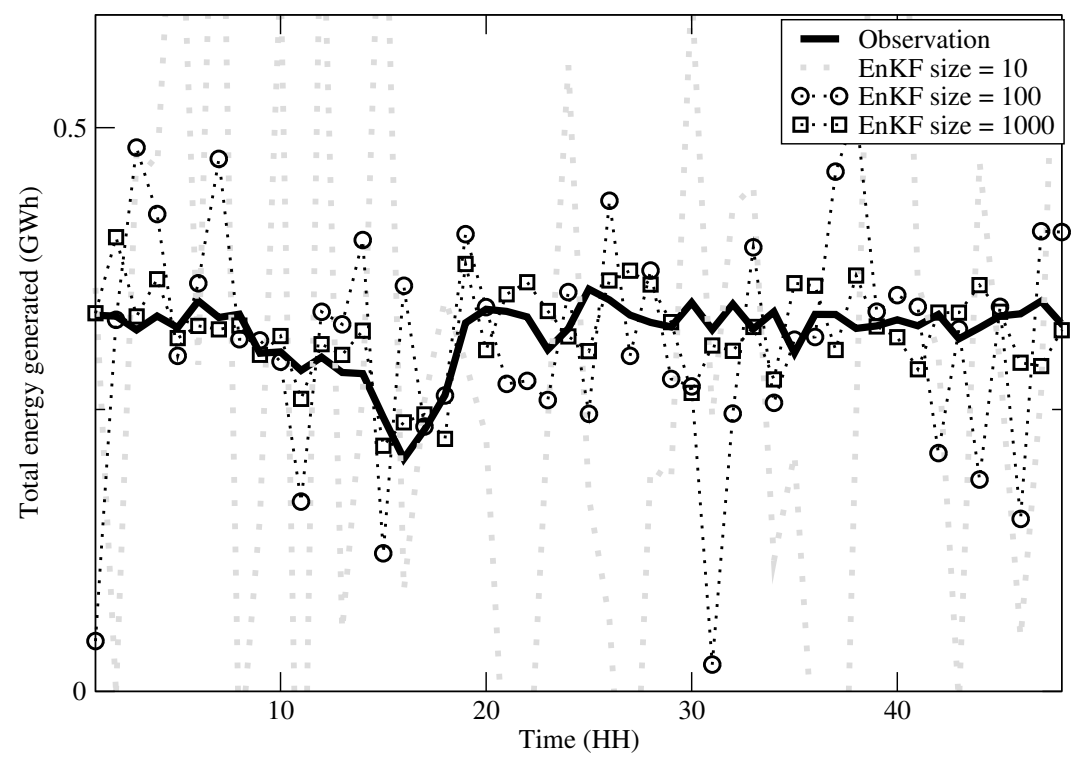

Figure 3: Wind-based energy generation with different EnKF realisations.

\subsection{EnOpt numerical simulation}

The resultant energy $Y^{u}$ is further used in the EnOpt algorithm in order to optimise the energy generation in the ED problem (Eqs. (9) and (20)) along with optimisation of controls $\mathbf{x}$ in Eq. (19)) following the imposed operational constraints in Eq. (13). The price penalty factor $h_{\max }$ is introduced that converts the emission function of the ED problem into the emission cost function.

Using the Monte-Carlo approach, the repeated random sampling of cost coefficients $\left(d_{i, t}^{\mathrm{G}}, d_{i, t}^{\mathcal{E}}\right.$ and $\left.d_{i, t}^{\mathrm{W}}\right)$ based on levelised costs from DECC [2012] are computed. The average $\mathrm{HH}$ bid-offer data for thermal units and the wind-generated energy are available in Elexon [2015], and this allows one to compute the bid-offer spread and updated cost coefficients using Eqs. (10) and (11). The cost coefficient of fuel and wind energy generation is shown 
in Table 1. Similarly, the emission cost coefficient is shown in Table 2.

\begin{tabular}{|c|c|}
\hline Cost coefficient & Cost $(£ / \mathrm{MWh})$ \\
\hline$d_{\mathrm{G}, 1, t}$ & {$[70,100]$} \\
$d_{\mathrm{G}, 2, t}$ & {$[120,135]$} \\
$d_{\mathrm{G}, 3, t}$ & {$[330,400]$} \\
$d_{\mathrm{G}, 4, t}$ & {$[70,75]$} \\
$d_{\mathrm{G}, 5, t}$ & {$[110,170]$} \\
$d_{\mathrm{W}, 1, t}$ & {$[133,138]$} \\
\hline
\end{tabular}

Table 1: Fuel and wind cost coefficient range.

\begin{tabular}{|c|c|}
\hline Cost coefficient & Emission cost $(£ / \mathrm{MWh})$ \\
\hline$d_{\mathcal{E}, 1, t}$ & {$[18,19]$} \\
$d_{\mathcal{E}, 2, t}$ & {$[5,42]$} \\
$d_{\mathcal{E}, 3, t}$ & {$[50,51]$} \\
$d_{\mathcal{E}, 4, t}$ & {$[0,5]$} \\
$d_{\mathcal{E}, 5, t}$ & {$[24,26]$} \\
\hline
\end{tabular}

Table 2: Emission cost coefficient range.

As mentioned, the time frame for the optimisation of costs is 24 hours and the control vector $\mathbf{x}$ is modified in every $\mathrm{HH}$ slot. The wind farm is assumed to be uncontrollable and therefore the generation output of the wind farm is balanced by those thermal units. The total number of control parameters for the vector $\mathbf{x}$ is $5 \times 48=240$, which is the product of the number of the thermal units and the number of the control steps. Total of 100 EnKF realisations are used in this example.

The maximum optimisation procedure is allowed to run for $\lambda_{\max }=200$ iterations. The iteration terminates when the relative increase of the EnOpt objective function is less than 1\%. As the steepest descent method is a type of unconstrained optimisation, the updated vector $\mathbf{x}$ which violates the control constraints (Eq. (13)) is truncated, and the new $\mathbf{x}$ is reallocated proportionally among generators based on the offset of truncated values.

The initial state of the control vector $\mathbf{x}$ is generated as the mean of the sampled lower and upper bounds of energy produced by the generating units. It is further perturbed with Gaussian random numbers $N \sim(0,0.5)$. Each component of $\mathbf{x}$ consists of energy generation value that corresponds to $i$ th generating units in Eq. (19) which is to be optimised. Quantity $\mathbf{x}$ is further integrated with $Y^{u}$ that optimises the energy generation. Total of five thermal units and one off-shore wind farm generator are considered in this EnOpt simulation. Constraints as outlined earlier in Eq. (13) are summarised in Table 3. '0' implies switchedoff generation. The TD losses account approximately $7.9 \%$ of the energy demand [DUKES, 2015].

The cumulative curves for the cost of generation for $\mathcal{C}_{B A U}$ and optimised $\mathcal{C}_{O}$ are shown in Fig. 4. The total $\mathcal{C}_{B A U}$ within the simulation run is obtained as $£ 12.16$ million, which is comparable to multiple simulation of generating units analysed by [Senthil and Manikandan, 2010, Subramanian and Ganesan, 2010]. The relative increases of cost savings based on $\mathcal{C}_{\mathrm{BAU}}$ and $\mathcal{C}_{\mathrm{O}}$ are shown in Fig. 5. It can be seen that the relative increase of the optimised costs of generation is relatively low within the time frame 0600-2000. This is due to the requirement 


\begin{tabular}{|c|c|c|}
\hline Notation & Description & Amount (GWh) \\
\hline$E_{\min , i}$ & Minimum allowable thermal generation capacity for: & \\
& $E_{\min , 1}$ & 67.20 \\
& $E_{\min , 2}$ & 100.80 \\
& $E_{\min , 3}$ & 0 \\
& $E_{\min , 4}$ & 84 \\
& $E_{\min , 5}$ & 0 \\
\hline$E_{\max , i}$ & Maximum allowable thermal generation capacity for: & 180 \\
& $E_{\max , 1}$ & 156 \\
& $E_{\max , 2}$ & 1.20 \\
& $E_{\max , 3}$ & 7.20 \\
& $E_{\max , 4}$ & 2.40 \\
\hline$E_{\mathrm{L}}$ & $E_{\max , 5}$ & 2.40 \\
\hline$E_{\mathrm{D}}$ & TD energy losses & 300 \\
\hline$W_{\max }$ & Energy demand & 36 \\
\hline
\end{tabular}

Table 3: Constraints of the ED problem.

to all generating plants to supply the required energy in response to the instant electricity market demand.

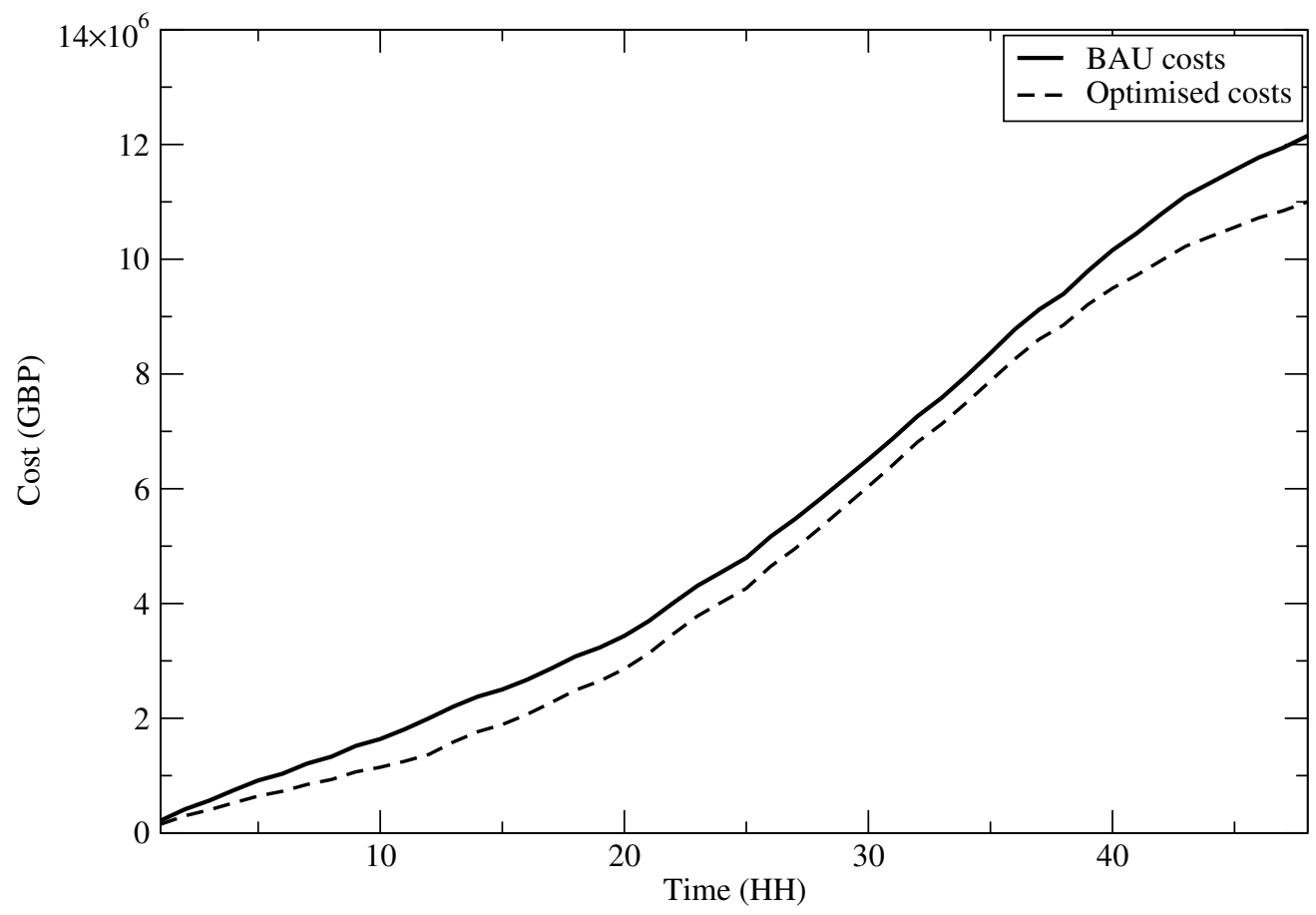

Figure 4: Daily cumulative plot of the business-as-usual versus optimised costs of the ED problem at every time step.

Carbon emissions plots of $\mathcal{E}_{B A U}$ and $\mathcal{E}_{O}$ are shown in Fig. 6. Due to the standardised regulations of costs in the electricity market, additional carbon emissions are produced in the afternoon. This shows the trade-offs between and emissions in the modern electricity 


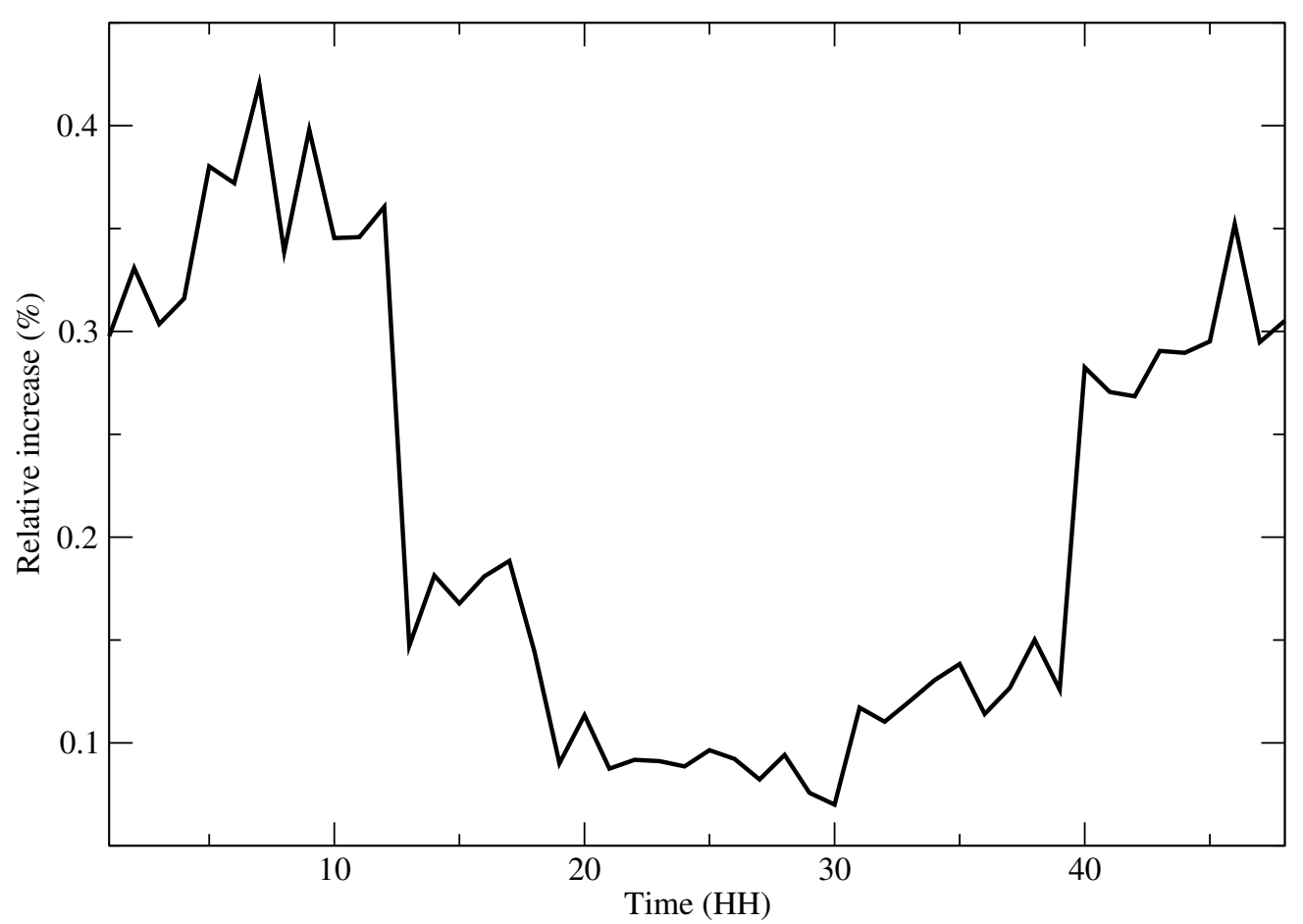

Figure 5: The relative increase of cost savings based on business-as-usual and the optimised at every time step.

market. Thus, the priority to optimise costs may lead to the increase of the emission level.

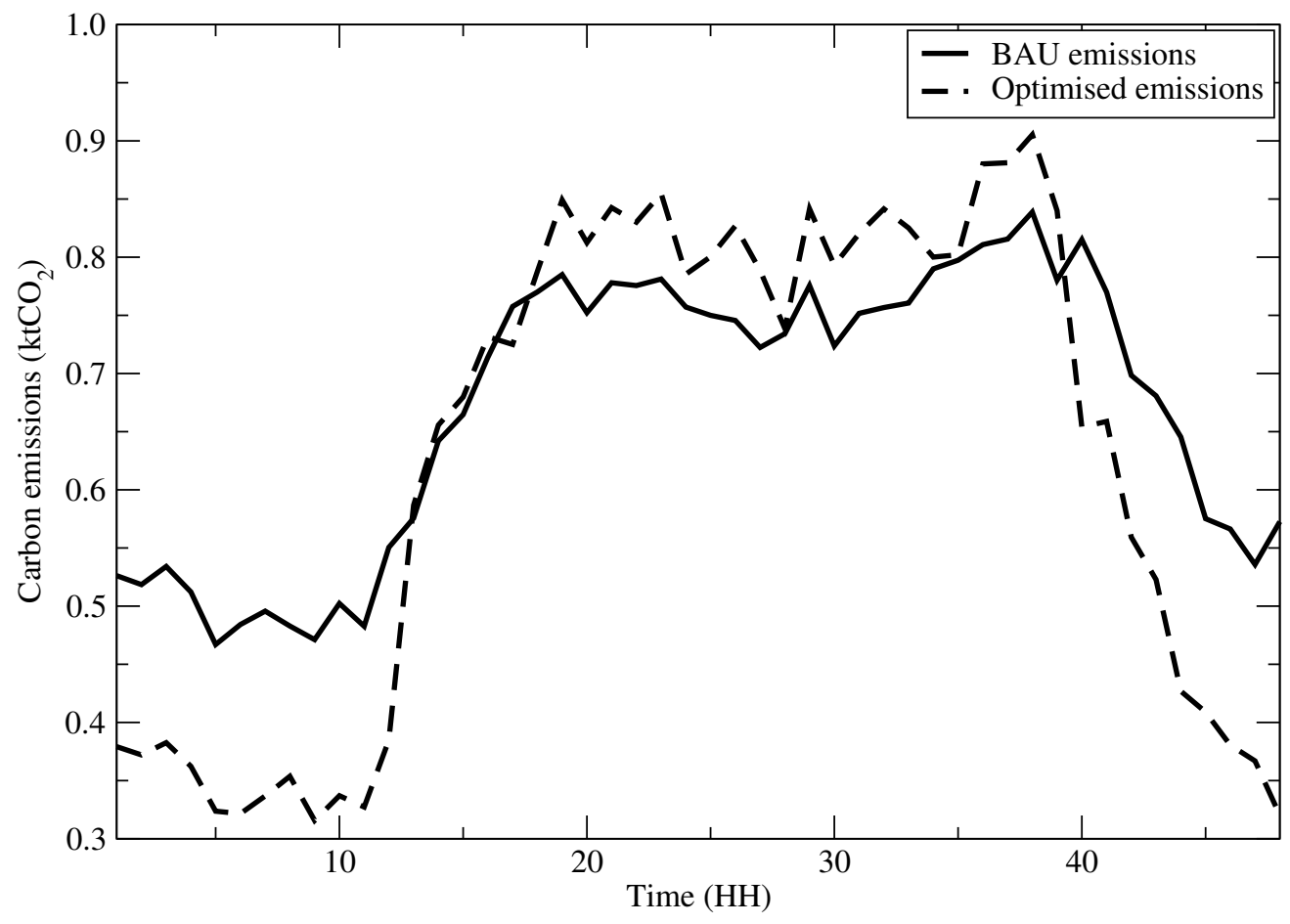

Figure 6: Carbon emissions for business-as-usual versus optimised in the ED problem at every HH time step. 


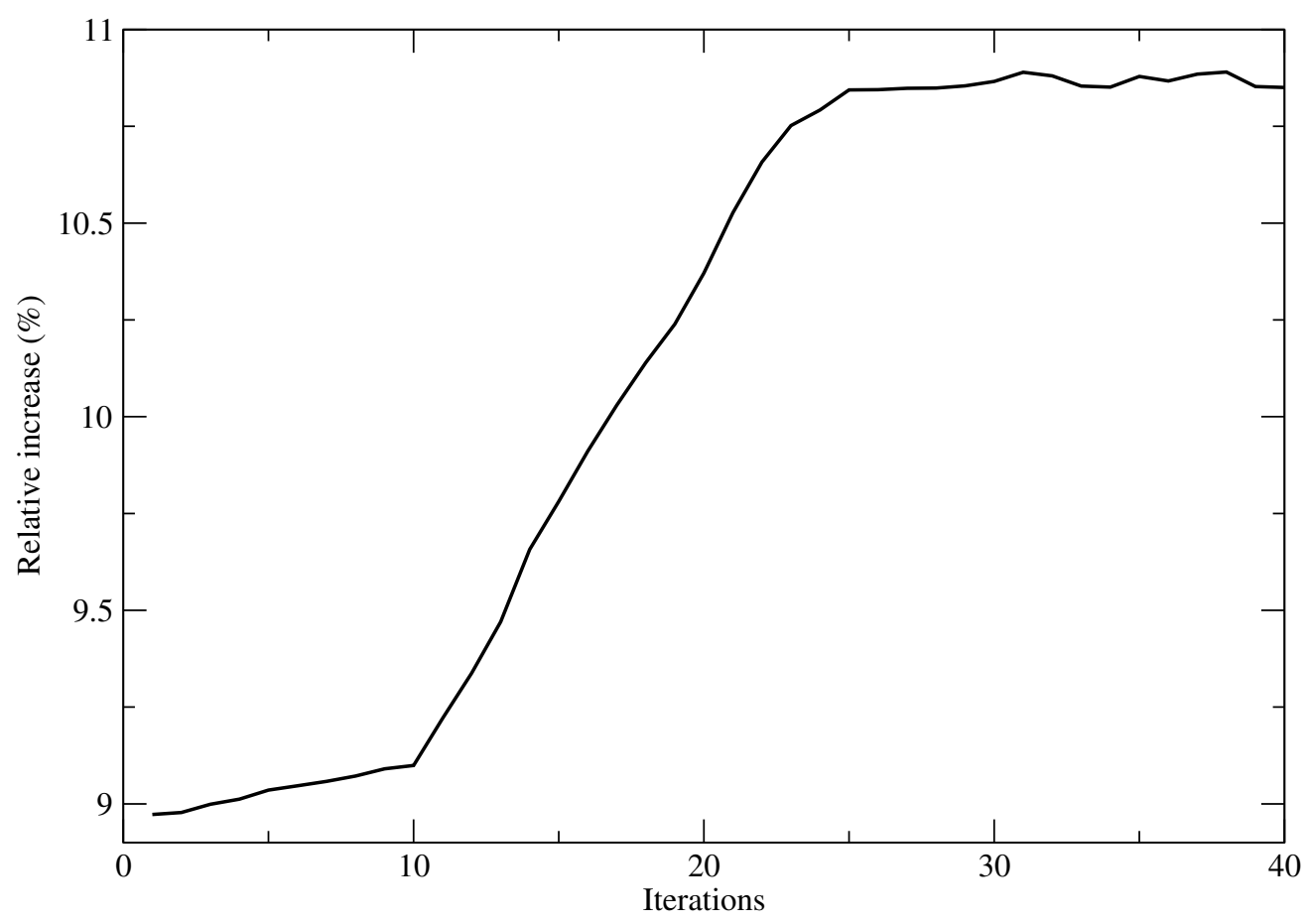

Figure 7: The relative increase $\left(\% \Delta_{\lambda, k}\right)$ of the objective function corresponding to $\lambda$ th iteration.

Finally, the relative increase $(\%)$ of $\mathcal{C}_{Y}\left(\mathbf{x}_{\lambda}\right)$ corresponding to $\lambda$ th iterations is shown in Fig. 7. We show the plot for 58 iterations in order to illustrate the clear trend of relative increase of $\mathcal{C}_{Y}\left(\mathbf{x}_{\lambda}\right)$, which converges at later iterations.

The optimised $\mathcal{C}_{O}$ at the end of EnOpt simulation (Eqs. (9) and (20)) are compared with $\mathcal{C}_{B A U}$ (Eq. (8)). Cost savings in this ED problem are calculated using Eq. (12) and are equal to $£ 1.15 \pm 0.10$ million. On the other hand, the second term of Eq. (9) is $\mathcal{E}_{O}$, which is used for estimation of carbon savings $\mathcal{E}_{S}$ in Eq. (2). Even though there are no carbon savings during the day time (based on Fig. 6), the overall savings $\mathcal{E}_{S}$ are achieved and are approximated as $0.81 \pm 0.1 \mathrm{ktCO}_{2}$ in one day of simulations.

Finally, the converged $\mathcal{C}_{Y}\left(\mathbf{x}_{\lambda}\right)$ indicates that $\mathbf{x}_{\lambda}$ is optimised. $\mathbf{x}_{\lambda}$ then propagates forward to regulate the amount of energy generation in the next time step.

\section{Conclusion}

In this paper, to address the increasingly used intermittent and uncontrollable wind-generated energy, other forms of generators are employed to balance the wind-generated energy outputs. EnOpt is used to optimise costs and carbon savings of generators in the power system that is subject to ED constraints, such as the generation capacity, electricity market balance and the actual wind generation limits. The established ED constraints also correspond to the current electricity market rules that maintain the security-off-supply in the power system.

Five energy generation profiles and one offshore wind generator from Elexon [2015], Balancing Mechanism Reporting Systems [2015] are forecast and assimilated using EnKF, and the assimilated data is further used in the EnOpt algorithm for the numerical optimisation. The parameters of the energy profile data are used in EnKF to sequentially predict and up- 
date the ensemble members and obtain filtered energy $y_{j}^{u}$. EnOpt optimises costs $\mathcal{C}_{O}$ using the updated $y_{j}^{u}$ assimilated from EnKF with optimal control $\mathbf{x}_{\lambda}$. At every time step of the EnOpt simulation, the resultant $\mathbf{x}_{\lambda}$ that optimises $\mathcal{C}_{Y}\left(\mathbf{x}_{\lambda}\right)$ is selected in order to maintain the current strategy in the ED problem with minimised costs and carbon emissions. The optimised carbon emissions can be also determined using the secondary term of the formulated objective function cost without incorporating the price penalty factor. At the end of the EnOpt simulation, $\mathcal{C}_{O}$ and $\mathcal{E}_{O}$ are collected to quantify $\mathcal{E}_{S}$ and $\mathcal{C}_{S}$.

Overall, EnKF demonstrates strength in minimising the mismatch between observations and EnKF updates, provided that the ensemble size must be sufficiently large: this improves convergence in EnKF propagation.

The ability to optimise $\mathcal{C}_{Y}\left(\mathbf{x}_{\lambda}\right)$ with convergence demonstrates the robustness of EnOpt. Optimal $\mathbf{x}_{\lambda}$ represents the electrical variables that may be incorporated in operational strategies in the power system that reduce costs and carbon emissions depending on the current demand.

Overall, the presented methodology is in line with the Capacity Market in UK Government's Electricity Market Reform (EMR) programme [DECC, 2016]. This is achieved by using the novel optimisation technique to ensure appropriate capacity of conventional generation in responding to intermittency of green generation. The EnOpt algorithm applied in this study is instrumental in regulating energy generation.

Additionally, due to random values of carbon intensity and cost coefficients at different time steps, uncertainties have been quantified in the simulations.

Already at the current stage of development of Smart technologies in the UK, the proposed methodology can be very useful for running and controlling complex power systems, accounting the emissions and renewable energy in a closed-loop optimal control. It can be used by aggregators, distribution network operators, and National Grid, under the regulations of the UK Office of Gas and Electricity Markets (Ofgem) requiring to quantify and minimise the trade-offs between and carbon emissions of the ED problem. The proposed routine provides an economical and environmental solution to allow business decisions based on both costs and environmental benefits of optimised energy generation.

The proposed framework is very general and combines both short-term forecast using EnKF and a flexible optimisation routine. Currently, the publicly available input required for applying the proposed methodology in real-time systems and control rooms is insufficient. Future analysis of real data provided by power network operators would expand applicability of the EnKF and EnOpt methods.

\section{References}

\section{References}

P. Albertos and A. Sala. Fuzzy logic controllers, advantages and drawbacks, September 1998. IEEE Transactions on Control System Technology.

R. Almendral-Vazquez and A. R. Syversveen. The ensemble Kalman filter - theory and applications in oil industry. Technical report, Norsk Regnesentral, 2006. [Online]. Available: https://www.nr.no/en/nrpublication?query=/file/4334/Almendral_ 
Vazquez_-_Ensemble_Kalman_Filter_-_theory_and_applications_i.pdf, [accessed 25.07.15].

M. U. Altaf, T. Butler, T. Mayo, X. Luo, C. Dawson, A. W. Heemink, and I. Hoteit. A comparison of ensemble Kalman filters for storm surge assimilation. Monthly Weather Review, 142:2899-2914, August 2014. doi: 10.1175/MWR-D-13-00266.1.

Balancing Mechanism Reporting Systems. BM reporting (updated daily). [Online]. Available: http://www. bmreports.com, 2015. [accessed 23.03.15].

N. Begum. Reservoir parameter estimation for reservoir simulation using ensembleK filter (EnKF). Master thesis, Department of Petroleum Engineering and Applied Geophysics, Norwegian University of Science and Technology, Trondheim, 2009.

Carbon Trust. Conversion factors. [Online]. Available: http://www.carbontrust. com/resources/guides/carbon-footprinting-and-reporting/conversion-factors, 2012. [accessed 16.05.15].

C. Chen, Y. P. Li, and G. H. Huang. Ensemble-based closed-loop optimization applied to Brugge field. SPE Reservoir Evaluation and Engineering, 13:72-81, February 2010a. doi: 10.2118/118926-PA.

C. Chen, Y. P. Li, and G. H. Huang. An inexact robust optimization method for supporting carbon dioxide emissions management in regional-power systems. Energy Economics, 40: 441-456, November 2013.

W. T. Chen, Y. P. Li, G. H. Huang, X. Chen, and Y. F. Li. A two-stage inexact-stochastic programming model for planning carbon dioxide emission trading under uncertainty. $A p$ plied Energy, 87(3):1033-1047, March 2010b.

Y. Chen, D. S. Oliver, and D. Zhang. Efficient ensemble-based close-loop production optimization. SPE Journal, 14(4):634-645, December 2009.

Convenant of Mayors. Technical annex to the seap template instructions document: the emission factors. [Online]. Available: http://www.eumayors.eu/IMG/pdf/technical_ annex_en.pdf, 2010. [accessed 08.07.13].

Mariesa L. Crow. Computational methods for electric power systems. CRC Press, New York, 2nd edition edition, August 2009. ISBN 9781420086607 - CAT\# 8660X.

T. Cui, H. Goudarzi, S. Hatami, S. Nazarian, and M. Pedram. Concurrent optimization of consumer's electrical energy bill and producer's power generation cost under a dynamic pricing model. In Proceedings of the Innovative Smart Grid Technologies (ISGT), pages 1-6, Washington, DC, 2012. IEEE PES.

DECC. Electricity generation costs 2013. Technical report, Department of Energy \& Climate Change (DECC), 2012. [Online]. Available: https ://www.gov.uk/government/uploads/ system/uploads/attachment_data/file/223940/DECC_Electricity_Generation_ Costs_for_publication_-_24_07_13.pdf, [accessed 31.07.15]. 
DECC. The UK 2050 calculator. [Online]. Available: http://2050-calculator-tool. decc.gov.uk/, 2013. [accessed 07.12.13].

DECC. Electricity Market Reform: Capacity Market. Department of Energy and Climate Change., [Online]. Available: https://www.gov.uk/government/collections/ electricity-market-reform-capacity-market, 2016. [accessed 25.03.16].

DEFRA. Defra greenhouse gas conversion factor repository. [Online]. Available: http: //www. ukconversionfactorscarbonsmart.co.uk, 2015. [accessed 17.05.15].

DG Clima. The 2050 low carbon economy. [Online]. Available: http://ec.europa.eu/ clima/policies/strategies/2050/index_en.htm, 2016. [accessed 17.02.16].

DUKES. Digest of united kingdom energy statistics (dukes) 2015: printed content. Technical Report ISBN 9780115155314, Department of Energy and Climate Change (DECC), 2015. Published with the permission of the DECC on behalf of the Controller of Her Majesty's Stationery Office.

Elexon. Imbalance pricing guidance. Elexon Ltd., [Online]. Available: https: //www. elexon.co.uk/wp-content/uploads/2014/11/imbalance_pricing_guidance_ v8.0.pdf, 2014. [accessed 31.07.15].

Elexon. Elexon portal. Elexon Ltd., [Online]. Available: https://www.elexonportal.co . $\mathrm{uk} /$ news/latest? cachebust=5tdys6qsn8, 2015. [accessed 02.08.15].

G. Evensen. Sequential data assimilation with a nonlinear quasi-geostrophic model using Monte-Carlo methods to forecast error statistics. Geophysical Research, 99(5):1014310162, May 1994.

G. Evensen. The ensemble Kalman filter: theoretical formulation and practical implementation. Ocean Dynamics, 53(4):343-367, November 2003.

K. Geetha, V. Sharmila Deve, and K. Keerthivasan. Design of economic dispatch model for Gencos with thermal and wind powered generators. Electrical Power and Energy Systems, 68:222-232, January 2015.

V. E. J. Haugen, G. Nævdal, L-J. Natvik, G. Evensen, A. M. Berg, and K. M. Flornes. History matching using the ensemble Kalman filter on a north sea field case. SPE Journal, 13(4):382-391, December 2008.

J. Hetzer, D. C. Yu, and K. Bhattarai. An economic dispatch model incorporating wind power. IEEE Transactions on Energy Conversion, 23(2):603-611, June 2008.

N. Hill, H. Venfield, C. Dun, and K. James. Government GHG conversion factors for company reporting, methodology paper for emission factors. Technical report, Department for Environment Food and Rural Affairs (DEFRA), 2013.

C.-M. Huang, S.-J. Chen, Y.-C. Huang, and H.-T. Yang. Comparative study of evolutionary computation methods for active-reactive power dispatch. IET Generation, Transmission E Distribution, 6(7):636-645, February 2012. 
Investopedia. How to calculate the bid-ask spread. Investopedia, LLC., [Online]. Available: http://www.investopedia.com/articles/investing/082213/ how-calculate-bidask-spread.asp, 2014. [accessed 02.08.15].

G. Jafroodi and D. Zhang. New method for reservoir characterization and optimization using crm-enopt approach. Journal of Petroleum Science and Engineering, 77(2):155-171, May 2011.

H. R. Jahangiri. Optimization of coupled $\mathrm{CO}_{2}$ sequestration and enhanced oil recovery. Phd thesis, Department of Petroleum Engineering, University of Southern California, Los Angeles, 2012.

J. P. Jensen. Ensemble Kalman filtering for state and parameter estimation on a reservoir model. Master thesis, Department of Engineering Cybernetics, Norwegian University of Science and Technology, Trondheim, 2007.

C. J. John and J. Mandel. A two-stage ensemble Kalman filter for smooth data assimilation. Environmental and Ecological Statistics, 15:101-110, March 2008.

N. A. Khan, A. B. Awan, A. Mahmood, S. Razzaq, A. Zafar, and G. A. S. Sidhu. Combined emission economic dispatch of power system including solar photo voltaic generation. Energy Conversion and Management, 92:82-91, January 2015.

E. T. Lau, Q. Yang, A. B. Forbes, P. Wright, and V. N. Livina. Modelling carbon emissions in electric systems. Energy Conversion and Management, 80(59):573-581, April 2014.

Y. F. Li, G. H. Huang, Y. P. Li, Y. Xu, and W. T. Chen. Regional-scale electric power system planning under uncertainty - a multistage interval-stochastic integer linear programming approach. Energy Policy, 38:475-490, January 2010.

Y. Z. Li, Q. H. Wu, and J. P. Zhan. Mean-variance model for power system economic dispatch with wind power integrated. Energy, 72:510-520, August 2014.

Q. G. Lin, G. H. Huang, B. Bass, Y. F. Huang, and X. D. Zhang. DESPU: Dynamic optimization for energy systems planning under uncertainty. Energy Sources, Part B: Economics, Planning and Policy, 6(4):321-338, July 2011.

G. Nævdal, L. M. Johnsen, S. I. Aanonsen, and E. H Vefring. Reservoir monitoring and continuous model updating using ensemble Kalman filter. In SPE Annual Technical Conference and Exhibition, pages 1-12, Denver, Colorado, USA, 2003. SPE.

A. Nemirovski, A. Juditsky, G. Lan, and A. Shapiro. Robust stochastic approximation approach to stochastic programming. SIAM Journal on Optimization, 19(4):1574-1609, January 2009.

J. Nwaozo. Dynamic optimization of a water flood reservoir. Master thesis, Mewbourne school of Petroleum and Geological Engineering, University of Oklahoma, Norman, 2006.

R. Petvipusit. Dynamic well scheduling and well type optimization using ensemble-based method (enopt). Master thesis, Mewbourne school of Petroleum and Geological Engineering, University of Oklahoma, Norman, 2011. 
POSTnote 268. Carbon factor of electricity generation. Technical report, Parliamentary Office of Science and Technology, 2006. [Online]. Available: http://www.parliament. uk/documents/post/postpn268.pdf, [accessed 01.10.14].

POSTnote 383. Carbon factor of electricity generation. Technical report, Parliamentary Office of Science and Technology, 2011. [Online]. Available: http://www.parliament.uk/documents/post/postpn_ 383-carbon-footprint-electricity-generation-references.pdf, [accessed 01.10.14].

S. Rajasomashekar and P. Aravindhababu. Biogeography based optimization technique for best compromise solution of economic emission dispatch. Swarm and Evolutionary Computation, 8:47-57, June 2012.

R. Ramanathan. Emission constrained economic dispatch. IEEE Transactions on Power Systems, 9(4):1994-2000, November 1994.

Narayan S. Rau. Optimization principles - practical applications to the operation and markets of the electric power industry. John Wiley \& Sons, Hoboken, New Jersey, September 2009. ISBN: 978-0-471-45130-4.

Ricardo-AEA. Ricardo-AEA: Home. [Online]. Available: http://www.ricardo-aea.com/ cms/, 2015. [accessed 03.10.15].

K. Senthil and K. Manikandan. Economic thermal power dispatch with emission constraint and valve point effect loading using improved tabu search algorithm. International Journal of Computer Applications, 3(9):6-11, July 2010.

N. Strachan and R. Kannan. Hybrid modelling of long-term carbon reduction scenarios for the uk. Energy Economics, 30(6):2947-2963, November 2008.

S. Subramanian and P. Ganesan. A simple approach for emission constrained economic dispatch problems. International Journal of Computer Applications, 8(11):39-45, October 2010 .

W. Wei, Y. Liang, F. Liu, S. Mei, and F. Tian. Taxing strategies for carbon emissions: a bi-level optimization approach. Energies, 7(4):2228-2245, April 2014.

Jizhong Zhu. Optimization of power system operation. John Wiley \& Sons, Hoboken, New Jersey, 2009. ISBN: 978-0-470-29888-6.

Y. Zhu, Y. P. Li, and G. H. Huang. Planning carbon emission trading for beijing's electric power systems under dual uncertainties. Renewable and Sustainable Energy Reviews, 41: 113-128, July 2013. 